\title{
Solvent-induced micelle formation in a hydrophobic interaction model
}

\author{
S. Moelbert, ${ }^{1}$ B. Normand ${ }^{2}$ and P. De Los Rios ${ }^{1}$ \\ ${ }^{1}$ Institut de théorie des phénomènes physiques, Ecole polytechnique fédérale de Lausanne, CH-1015 Lausanne, Switzerland \\ ${ }^{2}$ Département de Physique, Université de Fribourg, CH-1700 Fribourg, Switzerland
}

(Dated: October 30, 2018)

\begin{abstract}
We investigate the aggregation of amphiphilic molecules by adapting the two-state Muller-LeeGraziano model for water, in which a solvent-induced hydrophobic interaction is included implicitly. We study the formation of various types of micelle as a function of the distribution of hydrophobic regions at the molecular surface. Successive substitution of non-polar surfaces by polar ones demonstrates the influence of hydrophobicity on the upper and lower critical solution temperatures. Aggregates of lipid molecules, described by a refinement of the model in which a hydrophobic tail of variable length interacts with different numbers of water molecules, are stabilized as the length of the tail increases. We demonstrate that the essential features of micelle formation are primarily solvent-induced, and are explained within a model which focuses only on the alteration of water structure in the vicinity of the hydrophobic surface regions of amphiphiles in solution.
\end{abstract}

PACS numbers:

\section{INTRODUCTION}

In an aqueous solution, the hydrophobic parts of amphiphilic molecules tend to separate themselves from water molecules by forming aggregates, such as micelles and microemulsion droplets. The simplest amphiphilic structure occurs if the polar and hydrophobic parts of the amphiphilic molecule are well separated into head and tail regions. Motivated by the structure of sphingolipids and glycolipids (Fig. (1), we will refer to this type of molecule as 'lipids' (although we note here that this generic structure is not common to all classes of lipid). For molecules such as those in Fig. 11 micelles consist of a polar outer surface and a hydrophobic core which contains all the tails. By contrast, amphiphilic species whose polar and hydrophobic regions are distributed over the entire molecule, rather than being clearly separated, aggregate to form more general assemblies for the purpose of minimizing the hydrophobic area per molecule exposed to the aqueous phase. We will refer to this general category of mixed HP molecules as 'amphiphiles.' Poly(N-isopropylacrylamide), a polymer belonging to this class, exhibits a phase transition at a lower critical solution temperature (LCST) from a homogeneous solution, where the polymers are completely soluble, to a system of two separated phases 1]. At an upper critical solution temperature (UCST) the organic phase disaggregates, and above this temperature the amphiphilic molecules are again soluble due to entropic effects [2]. Substitution of polar by hydrophobic monomers in amphiphiles of given length leads to alterations of the critical solution temperatures which depend on the size of hydrophobic surface regions [2].

Micelle formation, and the aggregation of amphiphilic molecules in general, may be treated as a phase separation occurring at a critical micellar concentration (CMC) which describes the density of amphiphiles where the system enters the two-phase region [3, [4]. Above the CMC, amphiphilic molecules in aqueous solutions self-associate, forming small aggregates to decrease the net contact between their hydrophobic surfaces and the surrounding solvent. A small fraction of the lipid molecules remains free in the solution, with a concentration close to the $\mathrm{CMC}$ value. As the concentration increases, the onset of a semi-dilute regime is found, where the system may be considered as a solution of relatively few water molecules dissolved in an amphiphilic medium [5].

For lipid molecules, the CMC decreases as the length of the hydrophobic chain increases [6, 7, 8, 9], indicating a stabilization of aggregates as a consequence of the stronger net repulsion between the tail and the surrounding water. For the same reason, the LCST is thought to decrease as the tail grows, as suggested in Ref. 22]. One means of probing the nature of the phase diagram

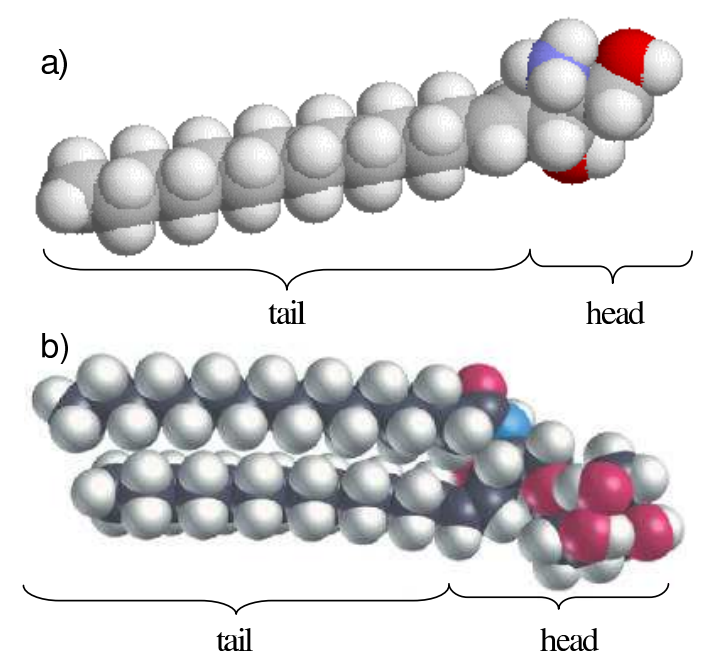

FIG. 1: Space-filling models of typical lipid molecules: (a) a sphingolipid (sphingosine, $\mathrm{C}_{18} \mathrm{H}_{37} \mathrm{O}_{2} \mathrm{~N}$ ), with one non-polar hydrocarbon tail; (b) a glycolipid $\left(\mathrm{C}_{40} \mathrm{H}_{72} \mathrm{O}_{8} \mathrm{~N}\right)$, with two tails. The relatively large, polar head-groups contain in addition oxygen and nitrogen atoms. 
and the effective hydrophobic interactions would be by systematic alteration of the polarity of the amphiphilic polymers in solution. Many theoretical and experimental studies have been conducted to describe the various aggregation phases of amphiphilic molecules in aqueous solutions [3, 4, 5, 6, 7, 8, 9, 10, 11, 12, 13, 14, 15, 16, 17, 18]. However, no comprehensive investigations have yet been performed concerning the influence of the distribution of polar groups in amphiphiles on the aggregation phase diagram, or concerning the mechanism underlying the process of self-aggregation.

The aim of this study is to substantiate experimental results indicating a decrease in LCST as the degree of hydrophobicity increases, and to analyze the dependence on density and hydrophobicity of the aggregation phase diagram. We will conclude that the principal properties of amphiphiles in aqueous solutions are solvent-induced, in that they are explained by alterations in the structure of liquid water in the vicinity of the hydrophobic regions of solute particles. We begin by introducing a simple hydrophobic-polar (HP) description (Sec. IIA) of amphiphilic solute particles (Sec. IIB) on a cubic lattice, and then extend the model to describe lipid particles of varying tail length (Sec. IC). In Sec. IIA we investigate the changes in the phase diagram associated with an increasing proportion of polar groups in amphiphilic molecules and with changes in their distribution. Sec. IIIB presents a similar analysis for the formation of lipid micelles in aqueous solutions. In Sec. IV we discuss the implications of our results and provide a brief conclusion.

\section{MODEL AND METHODS}

\section{A. Water Structure}

The solvation of amphiphiles in aqueous solutions and their self-association into micellar aggregates are genreally considered as a consequence of the effective hydrophobic interaction between polar water and the nonpolar regions of the solute molecules [19]. The unique properties of the aqueous medium which generate this interaction arise from the ability of water molecules to form strong hydrogen bonds both among themselves and with the polar groups of solute molecules. The formation and disruption of extended hydrogen-bonded networks leads directly to the delicate balance of enthalpic and entropic contributions to the solvent free energy which is responsible for the existence of a closed-loop aggregation regime in many solutions of polar molecules. This behavior is encapsulated in an adapted version of the model of Muller, Lee and Graziano (MLG) 20, 21], whose key features are as follows: consideration of a coarse-grained system whose sites contain clusters of water molecules of a size which matches that of the solute particles; a bimodal distribution of water clusters reflecting "ordered" sites with mostly intact hydrogen bonds, and "disordered" sites

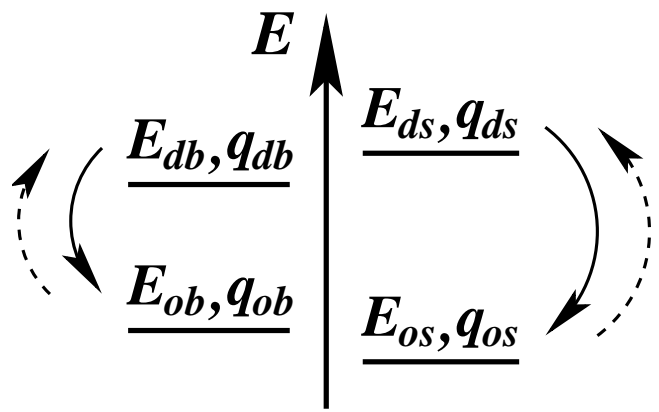

FIG. 2: Energy levels of a water site in the bimodal MLG model. The states are denoted $o s=$ ordered shell (cage conformation), $d s=$ disordered shell, $o b=$ ordered bulk, and $d b$ $=$ disordered bulk.

with relatively fewer intact hydrogen bonds; a further subdivision into "shell" sites neighboring the solute particles and "bulk" sites which are not disrupted by proximity to a polar surface; a set of microscopic energy and degeneracy parameters for these sites whose sequences are determined by the fact that breaking of hydrogen bonds increases site enthalpy but simultaneously raises the site degeneracy (number of orientational degrees of freedom of the water molecules), as represented in Fig. [2] Full details of the foundations and qualitative properties of the model can be found in Refs. 22] and [23].

The microscopic origin of the energy and degeneracy parameters for the four different types of water site (ordered/disordered and shell/bulk) in the adapted MLG model, based on both experimental [24, 25, 26] and theoretical 27, 28, 29, 30] analysis, is discussed in detail in Refs. 22, 31]. Here we highlight the competition of enthalpy and entropy terms using only the example of the ordered states. Although the insertion of a hydrophobic molecule leads to a destruction of local hydrogenbonding, at low temperatures the water molecules are found to rearrange in a cage-like structure around the solute molecule, which because of the orientational effect of the polar surface is formed by stronger hydrogen bonds. The consequent net energy reduction results in dissolution of the solute particle [24, 25, 26]. At higher temperatures, however, the additional entropic contributions available from the bulk solvent favor a minimization of local water restructuring, which drives the aggregation of hydrophobic solute particles to minimize their total surface exposed to water.

While it is possible to find perfect cages of hydrogen bonds for small hydrophobic particles, the formation of complete cages around large solute particles is prevented sterically. However, such large solute particles, and also their aggregates, may instead be surrounded by a number of partial cages, depending on their shape and surface roughness [29]. Atomic groups exposed at the surfaces of large molecules and aggregates present locally curved surface structures 32 which allow for a confined formation of partial cages. The lipid molecules we analyze (see 
Fig. 1) have a characteristic lengthscale of order $0.3 \mathrm{~nm}$ for the diameter of the chain and any side groups, 0.5 $\mathrm{nm}$ for the polar head, and a maximum length of 2-3 $\mathrm{nm}$ for the longer chains. The chain and head diameters are the lengthscales which determine the curvature of the molecule, and thus the possibility for cage formation. The considerations of the previous paragraph, specifically low-temperature solution, are then expected to remain valid for amphiphilic and lipid molecules, and this is confirmed by the success of models based on these concepts in describing large molecular species [33, 34, 35]. That the analysis is applicable for solute particles with a certain degree of curvature and surface roughness does not appear to be a significant restriction for molecules in the size range of most interest for amphiphilic and lipid characteristics.

Returning to the parameters of Fig. 2 we stress that the properties of the sysytem are critically dependent on the sequences $E_{d s}>E_{d b}>E_{o b}>E_{o s}$ of energy levels and $q_{d s}>q_{d b}>q_{o b}>q_{o s}$ of the corresponding degeneracies, which describe the entropy. These sequences are confirmed by a range of experimental measurements, and indeed if they are not maintained the solution does not exhibit a closed-loop aggregation regime. However, the results of the calculations to follow are not particularly sensitive to the exact values of the differences between these parameters, and are of course independent of their absolute values. We have used the energy values $E_{d s}=1.8, E_{d b}=1.0, E_{o b}=-1.0$, and $E_{o s}=-2.0$, which are thought to be qualitatively representative for aqueous solutions, and which have been successful in describing different types of solution 22, 23, 31, 35]. The respective degeneracies, normalized to a non-degenerate ordered shell conformation, are taken to be $q_{d s}=49, q_{d b}=40, q_{o b}=10$ and $q_{o s}=1$ [31]. These relative values have been found to be appropriate for reproducing the phenomenology of hydrophobic interactions 22, 31], protein denaturation [33, 34], swelling of biopolymers [35], and cosolvent effects on solubility of hydrophobic particles [23, 36]. Precise values of the microscopic parameters may in fact be refined by comparison with experimental measurements to yield semi-quantitative agreement for different solutions $[22,31,35,36$. The energy scale is correlated directly with a relative temperature scale, which we define as $k_{B} T \equiv \beta^{-1}$.

\section{B. Amphiphiles}

To include not only purely hydrophobic solute particles but also amphiphilic molecules with varying conformations of polar and non-polar regions, as in the experiments of Ref. [1], we represent the particles as cubes on which each face may be either polar or hydrophobic. A neighboring water site, which is homogeneously polar, interacts only with the side of the particle which is oriented in its direction. If this side represents a polar group, the neighboring water site is in a bulk state, whereas if the side is hydrophobic the water site is considered a shell state. Thus polar sides and water are considered as having the same effect on neighboring water molecules. However, polar faces do not contribute to the free energy. In this coarse-grained model, one side of a site may represent more than one chemical group, and thus corresponds to a net characterization of the surface area of the solute molecule under consideration.

Hydrophobic solute particles are generally larger than water, and thus a water site in the model consists of a group of molecules. On a lattice where each site has $z$ nearest neighbors, the energy of a system of $N$ sites, occupied either by amphiphiles $\left(n_{i}=0\right)$ or by water $\left(n_{i}=1\right)$, is given by the Potts-like Hamiltonian

$$
\begin{aligned}
& H\left[\left\{n_{i}\right\},\left\{\sigma_{i}\right\},\{k\}\right]= \\
& \quad \sum_{i=1}^{N} \frac{n_{i}}{z}\left[\left(E_{o s} \tilde{\delta}_{i, \sigma_{o s}}+E_{d s} \tilde{\delta}_{i, \sigma_{d s}}\right) \sum_{\langle j i\rangle}\left(1-\lambda_{i, j(k)}\right)\right. \\
& \left.\quad+\left(E_{o b} \tilde{\delta}_{i, \sigma_{o b}}+E_{d b} \tilde{\delta}_{i, \sigma_{d b}}\right) \sum_{\langle j i\rangle} \lambda_{i, j(k)}\right]
\end{aligned}
$$

where $\lambda_{i, j(k)}$ is a side variable depending on each of the nearest neighbors $j$ of site $i$, and takes the value 1 if the neighboring side of site $j$ is a polar side of a solute particle or water, and 0 otherwise. The variable $k$ takes values from 1 to $n_{c, j}$, where $n_{c, j}$ enumerates all equivalent orientations of site $j$. On a cubic lattice, the total number of sides of a solute particle is $z=6=N_{P}+N_{H}$, where $N_{H}$ is the number of hydrophobic cube faces. Thus for the cubic lattice $n_{c, j}=1$ for water and for solute particles with $N_{H}=6, n_{c, j}=6$ for $N_{H}=5, n_{c, j}=3$ for $N_{H}=4$ $\left(N_{H}=2\right)$ if the two polar (hydrophobic) sides are opposite to each other, $n_{c, j}=12$ for $N_{H}=4\left(N_{H}=2\right)$ if they are adjacent, $n_{c, j}=8$ for $N_{H}=3$ if the three polar sides are are all adjacent to each other, and $n_{c, j}=12$ for $N_{H}=3$ if two of them are opposite to each other.

Because a water side may be in one of $q$ different states, $\tilde{\delta}_{i, \sigma_{o s}}$ is 1 if it is in one of the $q_{o s}$ ordered shell states and 0 otherwise, and $\tilde{\delta}_{i, \sigma_{d s}}$ is 1 if it is in one of the $q_{d s}$ disordered shell states and 0 otherwise. Analogous considerations apply for the bulk states.

We note that for completely hydrophobic solute particles $\left(N_{P}=0\right)$ Eq. (11) is not equivalent to the Hamiltonian in Ref. 22], because the sites are treated differently. In Eq. (11) each face of a water site $i$ contributes to the free energy, and a sum is performed over all pairs of faces in contact with each other, as opposed to over individual sites. The representation in terms of cube sides is adopted here for consistent description of lipid molecules in Sec. IIC However, we will find in Sec. IIA that the qualitative differences between the two Hamiltonians for fully hydrophobic solute particles are small.

To determine the canonical partition function, a sum is performed over the state configurations $\left\{\sigma_{i}\right\}$. By taking 
into account the possible orientations of the amphiphilic particles through the variable $k$, the canonical partition function of the system of $N$ sites may be expressed as

$$
Z_{N}=\sum_{\left\{n_{i}\right\}} \prod_{i=1}^{N} \prod_{k=1}^{n_{c, j}} Z_{b}^{n_{i} \frac{1}{z} \sum_{\langle j i\rangle} \lambda_{i, j(k)}} Z_{s}^{n_{i} \frac{1}{z} \sum_{\langle j i\rangle}\left(1-\lambda_{i, j(k)}\right)}
$$

where $Z_{\sigma}=q_{o \sigma} e^{-\beta E_{o \sigma}}+q_{d \sigma} e^{-\beta E_{d \sigma}}$ for the shell $(\sigma \equiv s)$ and bulk $(\sigma \equiv b)$ states of pure water sides. The grand canonical partition function of the system for variable solute particle number is then

$$
\Xi=\sum_{N} e^{\beta \mu N_{w}} Z_{N}=\sum_{\left\{n_{i}\right\}} e^{-\beta H_{\mathrm{eff}}^{\mathrm{gc}}\left[\left\{n_{i}\right\}\right]},
$$

where $\mu$ represents the chemical potential associated with the insertion of water and $N_{w}$ denotes the number of water sites. Although the explicit terms of the model describe solely the states of water molecules in solution, it contains implicitly all multi-particle interactions between hydrophobic solute molecules [22].

The coexistence regions are characterized by measuring the UCST and the LCST for various numbers $N_{P}$ of polar sides per particle. To investigate variations in the effective hydrophobic interactions due to changes in polarity of the solute particles, we increase systematically the number of polar sides per particle and determine the coexistence region in each case.

The extent of aggregate formation in the system is determined from the number density of contacts between two hydrophobic cube sides, $n_{H-H}$, between two polar sides, $n_{P-P}$, and between a polar and a hydrophobic side, $n_{P-H}$. The number of contacts in a randomly distributed system with the same particle density $\rho_{p}$ is also calculated for comparison. In a random solution of solute particles with $N_{P}$ polar faces, whose positions and orientations are completely independent, these probabilities are given in the thermodynamic limit by

$$
\begin{aligned}
p(H-H) & =p(H)^{2}, \\
p(P-P) & =p(P)^{2}, \\
p(P-H) & =2 p(H) p(P),
\end{aligned}
$$

where $p(H)$ is the probability that a cube face is hydrophobic, $p(P)$ is the probability that it is polar, and by symmetry $p(H-P)=p(P-H)$. In a random system, the probabilities of occurrence of the different faces are independent of the neighboring sites, and are simply

$$
\begin{aligned}
p(H) & =\frac{N_{H}}{z} \rho_{p}, \\
p(P) & =\left(1-\rho_{p}\right)+\frac{N_{P}}{z} \rho_{p} .
\end{aligned}
$$

If the contact densities $n_{H-H}$ and $n_{P-P}$, are larger than their probabilities of random occurrence, and $n_{P-H}$, is correspondingly smaller, the system has formed aggregates which reduce the number of hydrophobic sides exposed to water.

\section{Lipids}

Micelles are generally formed by amphiphilic molecules, referred to here as lipids, which are composed of two distinct regions, the polar head and the hydrophobic tail. The length of the tail, which is typically composed of one or more hydrocarbon chains, is normally rather greater than the size of the polar head (Fig. 11). Experimental observations [38] suggest that lipids with tails shorter than 10 carbon atoms are highly soluble in aqueous solutions, while those whose tails exceed approximately 20 carbon atoms are almost completely insoluble. For those molecules with tail lengths in the intermediate range, which show the widest variety of surface-active properties, the tails are on average some three to five times longer than the dimensions of the polar head. (In lipids composed of two or more tails, this ratio is generally smaller.) As an example, the head size of the glycolipid in Fig. 11 is close to one third of the length of the 14-atom tail. We define an effective tail length $l$ as the ratio between the tail length and the head size of the lipid, so that a typical lipid is represented by $l$ values between two and five, which may also be fractional.

For simple geometrical reasons, the total repulsion between such a tail and the water molecules surrounding its sides is significantly stronger than that for the small tail tip of the chain. We adapt the model described in Sec. IB to include this aspect by assigning different energy levels to shell water clusters in contact with the sides of a solute particle compared to those in contact with the tip. Because the number of shell water molecules interacting with one side of a hydrophobic tail is approximately $l$ times that of those interacting with the tip, the energy associated with a site representing all of these water molecules is taken to be $l$ times that for a tip site. A cubic solute particle in the lipid model [represented in Fig. [10(a)] consists of a polar head (P) and a hydrophobic tail, which in turn is divided into the moderately hydrophobic tip $(\mathrm{H})$, situated opposite the polar head, and the long, strongly hydrophobic sides of the tail $(\mathrm{S})$. In the coarse-grained model, both the tip and each long side of the tail are represented by a face of the cubic particle, but the sides interact more strongly with a neighboring water site than does the tip.

The Hamiltonian of a system of $N$ sites on a cubic lattice, which are occupied either by water $\left(n_{i}=1\right)$ or by a lipid molecule $\left(n_{i}=0\right)$, is then

$$
\begin{aligned}
& H\left[\left\{n_{i}\right\},\left\{\sigma_{i}\right\},\{k\}\right]= \\
& \quad \sum_{i=1}^{N} n_{i}\left[\left(E_{o s, H} \tilde{\delta}_{i, \sigma_{o s, H}}+E_{d s, H} \tilde{\delta}_{i, \sigma_{d s, H}}\right) \frac{1}{z} \sum_{\langle j i\rangle}\left(1-\lambda_{i, j(k)}^{2}\right)\right. \\
& \quad+\left(E_{o s, S} \tilde{\delta}_{i, \sigma_{o s, S}}+E_{d s, S} \tilde{\delta}_{i, \sigma_{d s, S}}\right) \sum_{\langle j i\rangle} \frac{1}{2 z} \lambda_{i, j(k)}\left(1-\lambda_{i, j(k)}\right) \\
& \left.\quad+\left(E_{o b} \tilde{\delta}_{i, \sigma_{o b}}+E_{d b} \tilde{\delta}_{i, \sigma_{d b}}\right) \sum_{\langle j i\rangle} \frac{1}{2 z} \lambda_{i, j(k)}\left(1+\lambda_{i, j(k)}\right)\right],
\end{aligned}
$$


where again $\lambda_{i, j(k)}$ depends on the orientation state $k$ of the particle at each nearest-neighbor site $j$ of $i$. However, for the lipid model $\lambda_{i, j(k)}$ takes the value 1 if the neighboring face is polar (i.e. water or the relevant side of a solute particle), 0 if it represents a slightly hydrophobic tail tip $\mathrm{H}$, and -1 if it represents a strongly hydrophobic tail side S. $k$ varies again from 1 to $n_{c, j}$, where $n_{c, j}=1$ for water and $n_{c, j}=6$ for lipid particles. Because a polar side $i$ may be in one of $q$ different states, $\tilde{\delta}_{i, \sigma_{o s, H}}$ is 1 if site $i$ is occupied by a polar face in one of the $q_{o s}$ ordered shell states of a $\mathrm{H}$ face and 0 otherwise, and $\tilde{\delta}_{i, \sigma_{d s, H}}$ is 1 if it is occupied by pure water in one of the $q_{d s}$ disordered shell states of a $\mathrm{H}$ face and 0 otherwise. Analogous considerations apply for $\mathrm{S}$ faces and for the bulk states. We stress here that the Hamiltonians in Eqs. (1) and (6) provide a full description of the microscopic states of the amphiphilic and lipid systems. The macroscopic, thermodynamic behavior is contained implicitly in these microscopic states [20, 21, 22, 23, 31, 33, 34, 35, 36, 37], and the differences for such properties as the phase boundaries for varying chain length enter through the microscopic energy and degeneracy parameters.

As in Sec. [B only water sites contribute to the free energy, and a polar face is considered having the same effect on a neighboring water site as water. We define

$$
\begin{aligned}
\mathcal{S}_{H} & =-\frac{1}{\beta} \ln \left[q_{o s, H} e^{-\beta E_{o s, H}}+q_{d s, H} e^{-\beta E_{d s, H}}\right] \\
\mathcal{S}_{S} & =-\frac{1}{\beta} \ln \left[q_{o s, S} e^{-\beta E_{o s, S}}+q_{d s, S} e^{-\beta E_{d s, S}}\right] \\
\mathcal{B} & =-\frac{1}{\beta} \ln \left[q_{o b} e^{-\beta E_{o b}}+q_{d b} e^{-\beta E_{d b}}\right] .
\end{aligned}
$$

For a system of $N$ sites, the canonical partition function may be expressed as

$$
\begin{aligned}
Z_{N} & =\sum_{\left\{n_{i}\right\}} \prod_{i=1}^{N} \prod_{k=1}^{n_{c, j}} e^{-\beta \frac{n_{i}}{2 z} \mathcal{B} \sum_{\langle j i\rangle} \lambda_{i, j(k)}\left(\lambda_{i, j(k)}+1\right)} \\
& \times e^{-\beta \frac{n_{i}}{z}\left[\mathcal{S}_{H} \sum_{\langle j i\rangle}\left(1-\lambda_{i, j(k)}^{2}\right)+\mathcal{S}_{S} \sum_{\langle j i\rangle} \frac{1}{2} \lambda_{i, j(k)}\left(1-\lambda_{i, j(k)}\right)\right]}
\end{aligned}
$$

whence the grand canonical partition function for a system where the lipid density is not fixed is

$$
\Xi=\sum_{N} e^{-\beta \mu N_{w}} Z_{N}=\sum_{\left\{n_{i}\right\}} e^{-\beta H_{\mathrm{eff}}^{\mathrm{gc}}\left[\left\{n_{i}\right\}\right]},
$$

with the effective, grand canonical Hamiltonian

$$
\begin{aligned}
H_{\mathrm{eff}}^{\mathrm{gc}}\left[\left\{n_{i}\right\}\right]= & \sum_{i=1}^{N} \sum_{k=1}^{n_{c, j}}\left[n_{i}\left(\mathcal{S}_{H} \frac{1}{z} \sum_{\langle j i\rangle}\left(1-\lambda_{i, j(k)}^{2}\right)-\mu\right)\right. \\
& +\mathcal{B} n_{i} \sum_{\langle j i\rangle} \frac{1}{2 z} \lambda_{i, j(k)}\left(1+\lambda_{i, j(k)}\right) \\
& \left.+\mathcal{S}_{S} n_{i} \sum_{\langle j i\rangle} \frac{1}{2 z} \lambda_{i, j(k)}\left(1-\lambda_{i, j(k)}\right)\right] .
\end{aligned}
$$

To measure the formation of aggregates in the lipid system, the number of contacts between different faces is again determined and compared with the number of contacts in a randomly distributed system of the same particle density $\rho_{p}$. In a random solution of solute particles, whose positions and orientations are completely independent, these probabilities are given in the thermodynamic limit by

$$
\begin{aligned}
& p(\alpha-\alpha)=p(\alpha)^{2}, \\
& p(\alpha-\beta)=2 p(\alpha) p(\beta)=p(\beta-\alpha),
\end{aligned}
$$

where $\alpha, \beta \equiv H, P, S$. Here $p(H)$ is the probability that the adjacent side of the nearest neighbor is slightly hydrophobic, $p(P)$ the probability that it is polar, and $p(S)$ the probability that it is strongly hydrophobic. The probabilities are given in general by

$$
\begin{aligned}
& p(H)=\frac{N_{H}}{z} \rho_{p}, \\
& p(P)=\left(1-\rho_{p}\right)+\frac{N_{P}}{z} \rho_{p}, \\
& p(S)=\frac{N_{S}}{z} \rho_{p},
\end{aligned}
$$

although henceforth we will consider only the values $N_{H}=N_{P}=1, N_{S}=4$. Aggregate formation has occurred in this system if $n_{H-H}, n_{P-P}$, and $n_{S-S}$ are larger than their random expectation values, and $n_{P-H}$ and $n_{P-S}$ are correspondingly smaller.

The absolute energy levels and the degeneracies of water sites facing $\mathrm{S}$ sides are higher because this site represents the number of water molecules contained in a shell site of $\mathrm{H}$ multiplied by the factor $l$. The effective energy of a $\mathrm{S}$ shell site is thus obtained from that for a $\mathrm{H}$ shell site, for both ordered and disordered states, using

$$
E_{o s, S}=l E_{o s, H}, \quad E_{d s, S}=l E_{d s, H},
$$

where $E_{o s, H}\left(E_{d s, H}\right)$ is the energy of an ordered (disordered) water site in the shell of the tip $(\mathrm{H})$ and $E_{o s, S}$ $\left(E_{d s, S}\right)$ that of an ordered (disordered) shell water site of the tail side $(\mathrm{S})$. Under the assumption that neighboring water sites are rather independent, the total number of configurations for two sites may be approximated by the product of their numbers of configurations. Thus, the number of configurations of a $\mathrm{S}$ shell site is related to that of a corresponding $\mathrm{H}$ shell site by

$$
q_{o s, S}=q_{o s, H}^{l}, \quad q_{d s, S}=q_{d s, H}^{l} .
$$

The parameter values of the energy levels, and the degeneracies of bulk water and $\mathrm{H}$ shell sites, are chosen as described in Sec. IA

\section{Methods}

Our interest is focused on the orientation and location of amphiphilic molecules in solution, which may be captured by molecular-level simulations. We thus restrict 
our considerations to Monte Carlo studies, because the processes involved depend strongly on local, spatial effects which are neglected in mean-field calculations. As in Ref. 22], we use Monte Carlo simulations to detect the aggregation of amphiphilic solute particles as a function of temperature and solute concentration. We work with a system of $30 \times 30 \times 30$ sites on a cubic lattice with random initial particle distributions and periodic boundary conditions, using a Metropolis algorithm for sampling of the configuration space. The numbers of relaxation (100 000) and measurement (1000 000) steps are similar to those in our previous studies 22]. The closedloop coexistence curves in the $\rho_{p}-T$ phase diagram are obtained from the transitions determined by increasing the temperature at fixed chemical potential (grand canonical sampling), which results in a sudden density jump at the transition temperature, and from the corresponding solute particle densities.

Analysis of the properties of a lipid-water system at the molecular level is possible by similar Monte Carlo simulations using the model of Sec. IIC However, the procedure described above must be redefined in one respect. In the Metropolis algorithm, the relative transition probability to a configuration $\left\{n_{i}^{\prime}\right\}$ from a previous one $\left\{n_{i}\right\}$ depends on the difference in free energy of the two configurations according to

$$
r=e^{-\beta\left(H_{\mathrm{eff}}^{\mathrm{gc}}\left[\left\{n_{i}^{\prime}\right\}\right]-H_{\mathrm{eff}}^{\mathrm{gc}}\left[\left\{n_{i}\right\}\right]\right)} .
$$

The free-energy difference must be calculated for two states with the same number of molecules. One step of the simulation procedure consists either in rotation of a solute particle or in a site exchange between two randomly chosen sites. The only contributions to the difference in free energy are then the energy change of the sites concerned, and of their nearest-neighbor sides. If a side changes from $\mathrm{P}$ to $\mathrm{S}$ or vice versa, the bulk and shell states of neighboring water sites must contain the same number of water molecules to be comparable. In this case, we attribute the bulk energies $E_{o b, S}=l E_{o b}, E_{d b, S}=l E_{d b}$, and the respective degeneracies $q_{o b, S}=q_{o b}^{l}, q_{d b, S}=q_{d b}^{l}$, to the relevant water sites when calculating the probability $r$. The index $\mathrm{S}$ refers to the comparison of this bulk site with a $\mathrm{S}$ shell site.

\section{RESULTS}

\section{A. Amphiphiles}

We begin by attempting to capture the qualitative behavior of amphiphilic molecules with varying conformations of polar and non-polar regions. Fig. 3] shows the $\rho_{p}-T$ phase diagram obtained from the simple model of Sec. IB for different numbers of polar sides per particle. The densities represent volume fractions, and are therefore dimensionless. In the coexistence regime the amphiphilic particles aggregate, minimizing the contact

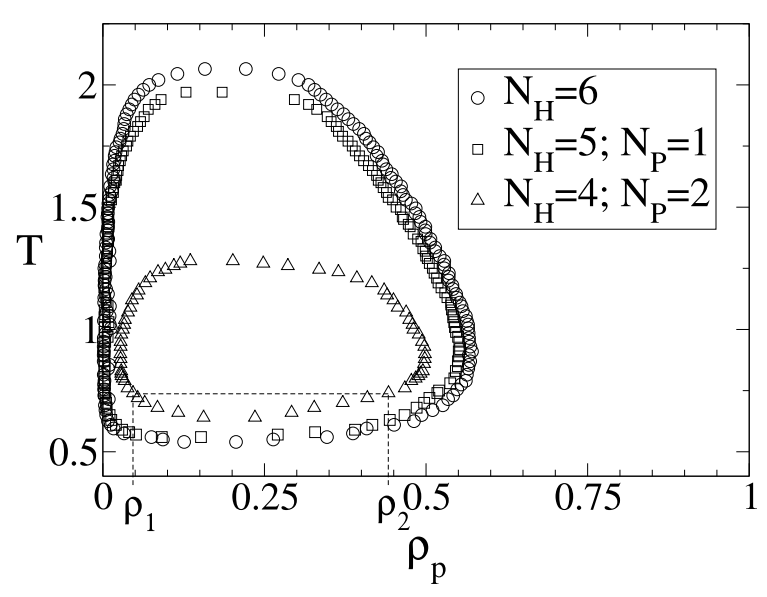

FIG. 3: $\rho_{p}-T$ phase diagram for micelle formation in the $3 \mathrm{D}$, HP model for different numbers of polar sides per solute particle. The coexistence region is reduced, and aggregation suppressed, as the number of hydrophobic sides substituted by polar sides increases. At a given dimensionless temperature $T_{L C S T}<T<T_{U C S T}$ the system is homogeneous for solute particle concentrations below the $\operatorname{CMC}\left(\rho_{1}\right)$, while above this value it separates into two phases of densities $\rho_{1}$ and $\rho_{2}$.

of their hydrophobic regions with water and with polar solute segments. Outside this region the amphiphiles are soluble at all densities below the LCST and above the UCST. For temperatures between these values, the amphiphiles are soluble only at very low densities, while at the $\mathrm{CMC}, \rho_{1}$, the particles aggregate and the system separates into two phases: nearly pure water (of particle density $\rho_{1}$ ) and an amphiphilic phase of density $\rho_{2}$. With increasing polarity, the solubility of the solute particles is enhanced and the CMC increases.

As expected, the coexistence region is reduced as the number of polar sides increases [2, 8]. If the system represents a solution of purely hydrophobic particles $\left(N_{P}=0\right.$ and $N_{H}=6$ ), the coexistence curve is almost identical to that found in the HP model in Ref. [22] (see Sec. [IB). Substitution of one hydrophobic side per particle by a polar side $\left(N_{P}=1\right.$ and $\left.N_{H}=5\right)$ leads to a decrease of the UCST and a slight increase of the LCST, and to a small overall suppression of the temperature and density range of the coexistence region. In this case, which may be taken to represent simplified lipid molecules (discussed in Sec. IV], the effect is rather moderate. However, substitution of a second hydrophobic side per particle $\left(N_{P}=2\right.$ and $\left.N_{H}=4\right)$ reduces the coexistence region dramatically (Fig. 3). When substituting three or more hydrophobic sides by polar ones, the solute particles become soluble at all temperatures and no aggregation phase transition is observed.

Figures 4 and 5]illustrate the nature of the aggregated phase using "snapshots" of two-dimensional (2D) systems at $T=1.0$, obtained in the coexistence region for solute particles with different numbers of polar sides. The 

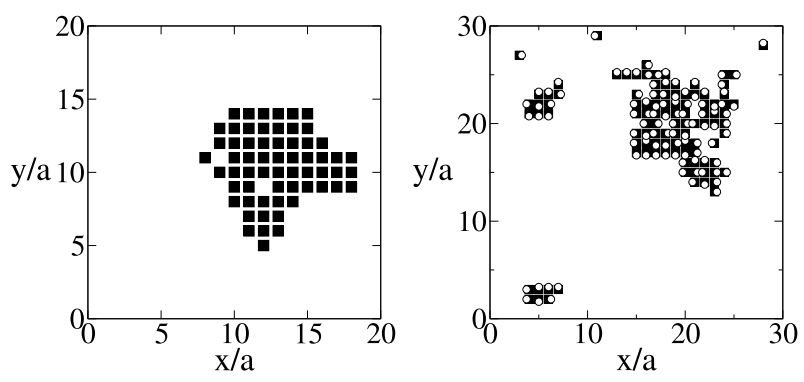

FIG. 4: Snapshots of 2D systems in the coexistence phase, obtained by Monte Carlo simulations at $T=1.0$. Left: completely hydrophobic solute particles $\left(N_{P}=0\right)$ in water; right: mainly hydrophobic solute particles $\left(N_{P}=1\right)$ in water. White circles (right) represent the polar sides of the solute particles, which are shown as black squares. The particles form compact micelles which shield the hydrophobic sides from water. The lattice constant $a$ is defined by the solute particle size.
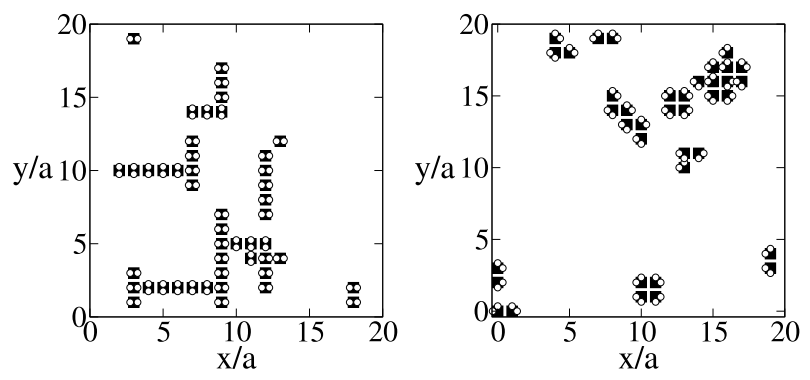

FIG. 5: Snapshots of 2D systems of partially hydrophobic solute particles $\left(N_{P}=2\right)$ in water in the coexistence phase, obtained by Monte Carlo simulations at $T=1.0$.

snapshots are taken after allowing the system to relax for 1000000 steps, where every $20^{t h}$ step is an attempt to exchange two sites and the others are attempts to rotate a particle. Systems containing primarily hydrophobic particles $\left(N_{P}=0\right.$ and $\left.N_{P}=1\right)$ form mostly compact clusters, which minimize the number of hydrophobic surfaces exposed to the solvent. For lipid-like solute particles with $N_{P}=1$ [Fig.6](a)], the formation of perfect micelles with a hydrophobic core and a polar surface is prevented by the nature of the square lattice, which causes frustration on the edges of the micelles: an edge particle is forced to expose one of its hydrophobic sides to water. The model allows a lipid particle to occur in the core of micelles, where its polar side is in direct contact with the polar side of another lipid particle, because no distinction is made between a group of water molecules and the polar face of a particle. Incorporating this distinction into the description could be expected to generate more realistic micellar structures, albeit within the confines of the cubic geometry.

Amphiphilic solute particles whose surface is half polar a)

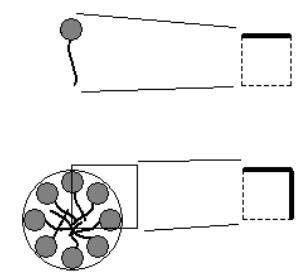

c)

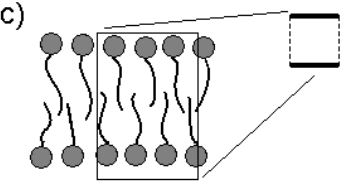

FIG. 6: Illustration of schematic analogs obtained using square particles with different arrangements of polar sides in 2D (see text). Hydrophobic sides of the square solute sites are shown as thick solid lines, polar sides as dashed lines.

$\left(N_{P}=N_{H}=2\right.$ in $\left.2 \mathrm{D}\right)$ show differing behavior depending on the polarity pattern of the sides. If the polar sides are adjacent on the square, small micelles consisting of four solute particles can be formed, which is energetically the most favorable configuration because no hydrophobic sides are exposed to water (Fig. [5). Short, diagonal lines of molecules, which may be considered to represent condensed bilayers, can also be formed, although their ends are hydrophobic, and this configuration is therefore less favorable than are "circular" micelles of four solute particles. These configurations are expected from the construction of the sites, which is shown in Fig. [6 to appear as the ground states. Solute sites with two adjacent polar sides may be considered to represent sections of circular micelles [Fig. [6)], with the formation of small micelles as a consequence. In contrast, if the polar sides are opposite each other, the only possibility to avoid hydrophobic sides being in contact with water is to form lines of particles, although again the hydrophobic ends remain exposed to water. These squares may be taken as schematic representations of cross-sections of a bilayer [Fig. [6(c)].

The contact densities $n_{H-H}, n_{P-P}$, and $n_{P-H}$, are shown in Fig. 7 for a system of solute particles with one polar side $\left(N_{P}=1\right)$ as a function of relative temperature. The results are normalized to the probability of these contacts in a randomly distributed system with the same particle density, $\rho_{p}=0.24$. At low temperatures, the solute particles are clearly soluble, because $n_{H-H}<p(H-H), n_{P-P}<p(P-P)$, and $n_{P-H}>p(P-H)$. This reflects the formation of strongly hydrogen-bonded, partial cage-like structures of water molecules around the hydrophobic parts of the amphiphilic particles when entropy effects are minor. At temperatures higher than the lower critical temperature for density $\rho_{p}$, increasing entropy effects favor a screening of hydrophobic faces from polar ones, and the solute particles aggregate to form micelles. In this regime $n_{H-H}>p(H-H), n_{P-P}>p(P-P)$, and the density of polar-hydrophobic contacts is suppressed, $n_{P-H}<p(P-H)$. The effect on $n_{H-H} / p(H-H)$ is more pronounced than $n_{P-P} / p(P-P)$ because the number of polar sides in the system is higher than the number of hydrophobic ones. Changes in $n_{H-H}$ then lead to a larger relative effect, and in fact the majority of $P-P$ contacts 


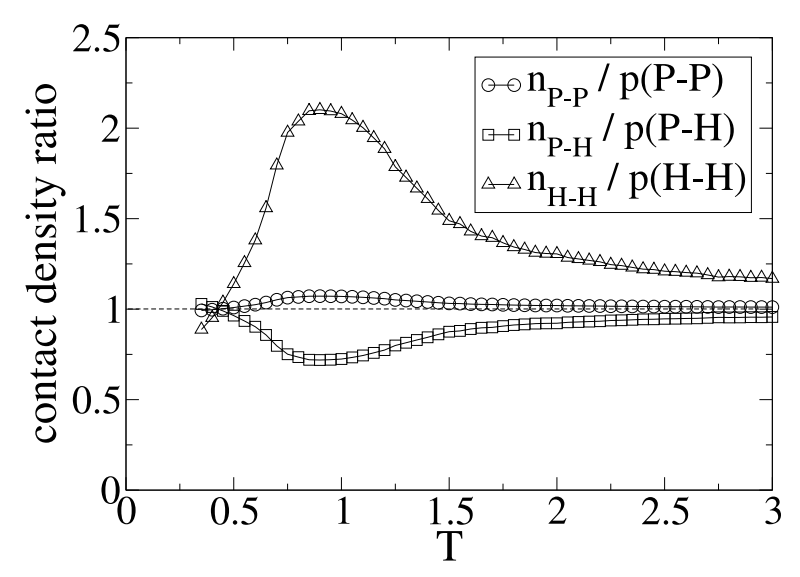

FIG. 7: Contact densities between polar and hydrophobic sides as a function of dimensionless temperature for a $3 \mathrm{D}$ system of solute particles with one polar side, $N_{P}=1$. The contact densities between sides are normalized to the values expected in a random system for $\rho_{p}=0.24$.

are intact even in the dissolved phase due to the high number of water molecules. For low particle densities, the relative effect is larger still because fewer $H-H$ contacts are possible.

Finally, at high temperatures, the entropy becomes dominant and the contact densities approach their respective random values as complete mixing is obtained. We note that at high temperatures the contact densities do not recross the value 1 to recover the low-temperature phase of single-particle dissolution (Fig. [7). Instead their values simply converge to unity, implying that the complete miscibility takes the form of a truly random particle/water distribution.

\section{B. Lipids}

We have performed Monte Carlo simulations for lipid molecules in water on a cubic lattice for varying lengths $l$ of the hydrophobic tail, as described in Secs. ПIC and IID to investigate the stability of their aggregation as a function of hydrophobicity $(l)$ and density. The $\rho_{p^{-}}$ $T$ phase diagram (Fig. 8) shows clearly that the coexistence regime is enhanced significantly with increasing tail length $l$, which can be attributed to the stronger effective repulsive interaction between the longer hydrophobic tail of the lipid molecule and the surrounding water. The ratio $l$ can be taken as the important quantity to characterize the degree of hydrophobicity of a lipid molecule. Comparison with Fig. 11indicates that for a typical polar head, $l=1$ corresponds to a tail containing approximately four carbon atoms. Fig. 8 shows that for lipid molecules with a tail containing approximately 8 carbon atoms $(l=2)$ there is already an enhancement of the

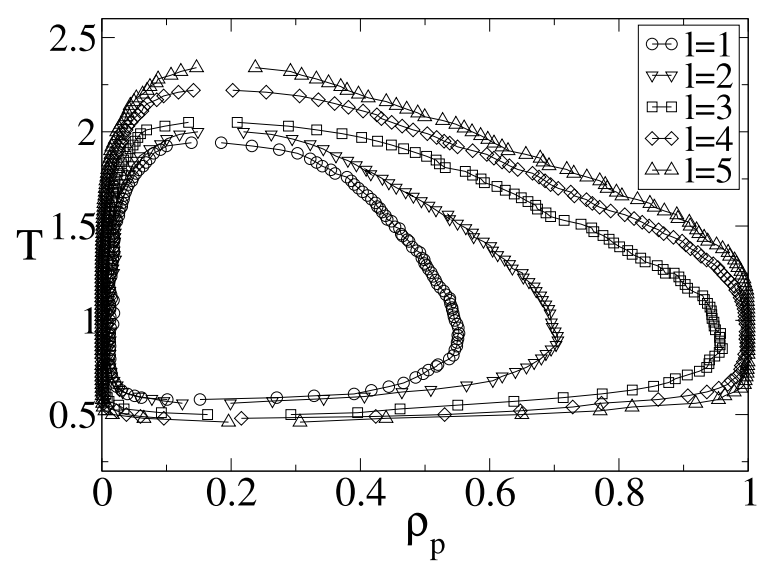

FIG. 8: $\rho_{p}-T$ phase diagram illustrating micelle formation in the extended 3D, HP model for lipid molecules of varying tail length. $l$ represents the relative length of the hydrophobic tail compared to the size of the head (Fig. 1). The coexistence region is enhanced as the length of the hydrophobic tails increases, and aggregation is promoted.

coexistence region. The aggregation of solute particles with a tail composed of approximately 12 carbon atoms $(l=3)$ is reinforced very significantly. Within our simplified model, at relative temperature $T=1$ lipids with $l \geq 4$ are basically insoluble, forming a completely separated phase, and thus perfect micelles, for all densities, in rather good qualitative agreement with expectations based on experiments 38 ].

The aggregation may be analyzed in more detail by studying the density of side contacts as a function of temperature. The thermal evolution of the density of contacts between the different sides in a system of lipid particles of tail length $l=3$ in water is shown in Fig. 9 The results are normalized to the corresponding probability of these contacts in a randomly distributed system with the same particle density, $\rho_{p}=0.25$. At low temperatures $n_{H-H}<p(H-H), n_{P-P}<p(P-P)$, and $n_{S-S}<p(S-S)$, while $n_{P-H}>p(P-H), n_{P-S}>p(P-S)$, and $n_{H-S}>p(H-S)$, meaning that the solute particles are clearly dissolved as a consequence of partial cage formation around the hydrophobic tails. At temperatures higher than the lower critical temperature for density $\rho_{p}$ (Fig. 8), the lipid particles aggregate to minimize their total exposed surface, whence $n_{H-H}>p(H-H), n_{P-P}>$ $p(P-P)$, and $n_{S-S}>p(S-S)$, while $n_{P-H}<p(P-H)$ and $n_{P-S}<p(P-S)$. Because of the much stronger effect of an $\mathrm{S}$ face than of an $\mathrm{H}$ face on its neighboring water site, the normalized contact density of two $\mathrm{S}$ faces is highest in the aggregation phase and lowest below the LCST. A higher relative contact density is observed between $\mathrm{S}$ and $\mathrm{H}$ faces than between two $\mathrm{H}$ faces for the same reason, while below the LCST water sites prefer to form the solvation shell of $\mathrm{S}$ faces rather than of $\mathrm{H}$ faces. 


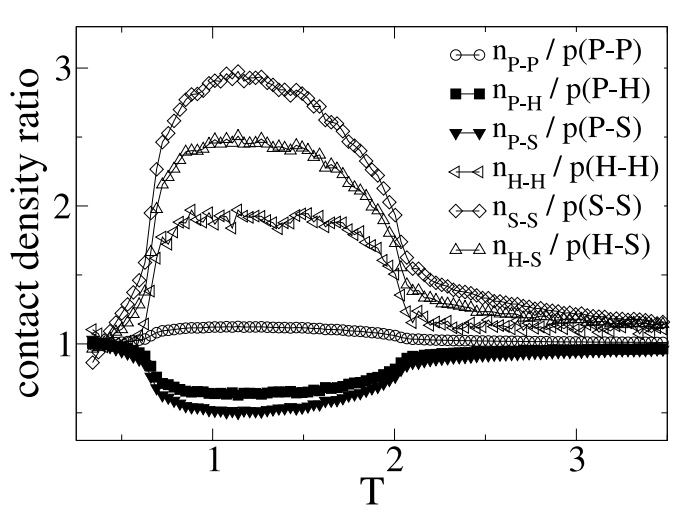

FIG. 9: Contact densities between the different faces as a function of dimensionless temperature for a $3 \mathrm{D}$ solution of lipid molecules with effective tail length $l=3$. The contact densities between sides are normalized to the values expected in a random system for $\rho_{p}=0.25$.

At high temperatures the contact densities converge, in fact rather abruptly (Fig. 9) to their random values, indicating complete mixing.

\section{DISCUSSION}

Amphiphilic molecules in aqueous solutions can form different types of micelles depending on their concentration and on the distribution of polar regions at their surfaces. Our initial investigation of the qualitative properties of micelle formation in a hydrophobic-polar model involved systematic substitution of the hydrophobic sides of cubic solute particles by polar ones. We determined the $\rho_{p}-T$ phase diagram for different surface patterns and found closed-loop coexistence curves (Fig. 3), in accord with experiments using hydrophilically modified copolymers of poly(N-isopropylacrylamide) 2]. With increasing polarity, the coexistence region is reduced as the solubility of the model amphiphiles increases. This tendency was confirmed by the same experiment, where the LCST of purely hydrophobic poly $(\mathrm{N}-$ isopropylacrylamide) P3 was observed to increase from $37^{\circ} \mathrm{C}$ at atmospheric pressure to $42-44^{\circ} \mathrm{C}$ when approximately $13 \%$ of the monomers were substituted by polar species (CP2 and CP3). In our model we observe the same quantitative increase of $2-3 \%$ in absolute temperature from $T_{L C S T}=0.545$ for purely hydrophobic solute particles to $T_{L C S T}=0.56$ for amphiphiles with one polar side, which represents $17 \%$ of the particle surface. Thus the crude cubic model appears to yield good agreement with available data at this level of comparison.

For particles with two polar sides the coexistence region is reduced dramatically. This is not surprising, considering the fact that two polar sides represent one third of the total particle surface, and the attractive in- a)

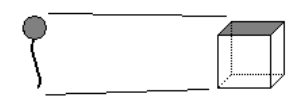

b)

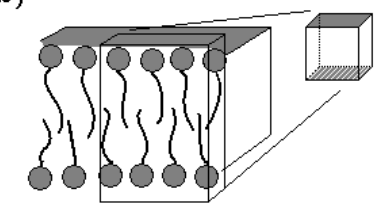

c)

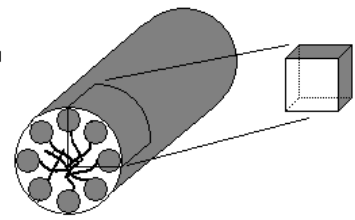

d)

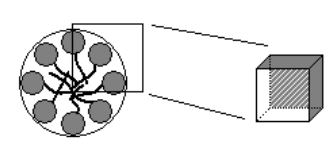

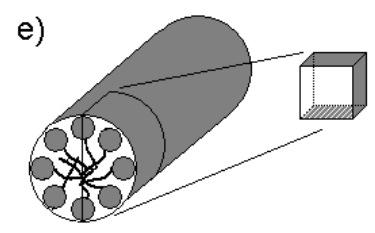

FIG. 10: Representation of different surface patterns on cubic solute particles in the HP model, and their schematic correspondence to different micelle types(see text). Polar surfaces and micelle segments are marked in gray, hydrophobic surfaces in white.

teractions with water are rather strong. In amphiphilic molecules the polar region is usually rather small compared with the hydrophobic surface area. The solubility of the molecules thus increases considerably on substitution with polar monomers, leading to a decrease in UCST and an increase in LCST and CMC. In the cubic model no significant difference in the size of the coexistence region is observed for different distributions of the two sides on the solute cubes. Any further substitution of hydrophobic faces by polar ones results in molecules which are at least half polar: the solubility of such particles is always high, and thus no aggregation is found.

Possible schematic interpretations of the various surface patterns of cubes representing solute particles in the HP model are shown in Fig. 10] The model is applicable for any surface pattern and density of solute, with the premise that one site may contain one amphiphilic molecule or a group of solute molecules. A given surface distribution of polar groups on a polymer may be characterized by a corresponding arrangement of polar sides on the surface of a cubic solute molecule. A single lipid molecule with a clear distinction between a polar head and a hydrophobic tail may be represented by a cube with one polar face $\left[N_{P}=1\right.$, Fig. [10(a)]. A small section of a cylindrical micelle would be represented by a cube with two adjacent polar sides $\left[N_{P}=2\right.$, Fig. 10(c)], while a particle with two opposite polar sides corresponds at the same level of approximation to a cross-section of a bilayer [Fig. 10(b)]. For the formation of "spherical" micelles, each site must have three adjacent polar sides $\left[N_{P}=3\right.$, Fig. [10 (d)]; if two of the three sides are situated opposite each other [Fig. [10(e)], the site may again be considered as a section of a cylindrical micelle. Although by construction $N_{P}=3$ should give small spherical or cylindrical micelles, depending on the distribution pat- 
tern at the surface of each site, in fact the solubility is too high to find aggregates.

Micellar structures occurring in 3D simulations are difficult to display. To confirm the formation of different micelle types depending on the surface pattern of the solute particles, we have considered snapshots of an analogous 2D system (Figs. 4 and 5). Here the square solute particles may be interpreted in a manner similar to the 3D case (Fig. 6), and the formation of small micelles and layers is found in the coexistence region. During the relaxation process, micelles grow from initial dimers to larger entities. Although the solute particles may rotate at a given position, they can be trapped in a configuration which disables the construction of perfect micelles or extended layers. Because there is no preference for growing a layer in one direction rather than in the other, short line segments are formed which are incompatible with others, resulting in a network of short layers. In the model, no distinction is made between the polar side of a water molecule and that of a solute particle, and a $P-P$ contact contributes the same energy independent of the molecules to which the sides under consideration belong. Such contacts between the polar sides of solute molecules are found in the interior of a micelle, which also influences the formation of perfect micelles. The non-zero temperature in the coexistence region, and the observation that the upper critical density is much smaller than unity, might further imply the formation of imperfect micelles. In fact the extent to which the upper critical density is significant remains unclear, because shell water sites in the model may be considered as belonging to the micelle phase rather than to the pure water phase, which would explain the low density of the organic phase even for perfect micelles containing no water molecules.

We have extended our analysis to describe lipids, which represent a particular type of amphiphilic molecule. Lipids are distinguished by a special partition of the polar and hydrophobic segments along the molecule: a typical lipid molecule consists of a polar head and one or two hydrophobic tails. To incorporate these geometrical features in the model we have adapted the energy levels and their degeneracies according to the tail length $l$, where a typical lipid molecules exhibiting surface-active properties would be represented by values $2 \leq l \leq 5$ [38].

As expected from experiments [8], Monte Carlo simulations of lipids of increasing length illustrate a significant enhancement of the coexistence region (Fig. 8). Lipids with a longer tail have more pronounced characteristics of hydrophobic solute particles than do lipids where the polar head represents a considerable fraction of the molecule. The solubility of long-tailed lipids is therefore lower than that of short-tailed ones, causing a decrease in LCST, an increase in UCST, and a decreased CMC at any given temperature as the tail becomes longer. For the parameters used in the model we find that an increase from $l=1$ to $l=2$ already enhances the coexistence region substantially, and for that $l \geq 4$ the system is completely separated into pure water and essentially perfect micelles of density $\rho_{p}=1$ over a wide temperature range, which is fully consistent with experiment [38].

A quantitative measure of micelle formation in amphiphilic and lipid systems is provided by the contact densities $n_{\alpha-\alpha}$ and $n_{\alpha-\beta}$, normalized by the corresponding probabilities of the contacts in a random system (Figs. 7 9). We have found fewer contacts between hydrophobic faces and more hydrophobic-polar contacts than would be expected for a random distribution below the LCST, indicating highly dissolved solute molecules. Above the LCST this picture is inverted, thus confirming the aggregation of amphiphiles and lipids, and the separation of the solution into two phases. From a knowledge of the system geometry and densities, the values of the contact ratios may also be used to confirm the extent of solution or aggregation, and also the effective "purity" of the micelles which form for different polar distributions on the solute molecules. At temperatures in excess of the UCST, the solution approaches a random mixture due to dominant entropy effects; in Monte Carlo studies the relative contact densities approach unity continuously, rather than undergoing a sharp transition.

We close by emphasizing again the limits of our analysis. We have formulated a model for an aqueous solution using a minimal set of assumptions; we have used an extremely crude representation of amphiphilic molecules, and have considered only a cubic system as the foundation on which our "micelles" are constrained to form. Nevertheless, we have obtained a realistic set of aggregation phenomena and a surprising degree of agreement with the available experimental results. There are, however, several examples of phenomena which are beyond the reach of the model at its current level of refinement. A solution of lipids in water may produce a lamellar phase of bilayers at low temperature and rather high densities [15]. Bilayer formation requires the possibility of smooth curvature and high flexibility [5], and is precluded in the Monte Carlo simulations by geometrical constraints presented by the lattice. For the same geometrical reasons it is also difficult to find well-formed micelles in the more general case of amphiphiles in water.

A further limitation is that the model considers explicitly the energy states of water sites, and therefore no distinction is made among $\mathrm{P}-\mathrm{P}, \mathrm{H}-\mathrm{H}, \mathrm{S}-\mathrm{H}$, and $\mathrm{S}-\mathrm{S}$ contacts. Because there is also no difference between a group of water molecules and the polar face of a solute particle, the model a llows amphiphiles to occur in the core of micelles, where their polar sides are in direct contact with those of other amphiphiles. For lipids, an S-H contact which is formed during the Monte Carlo simulation is as favorable as an S-S contact, although it is more likely to be broken in a later step. In addition, the orientation of a lipid molecule is irrelevant for the formation of an S-S contact, which prevents efficient alignment of the heads and may further hinder the formation of extended bilayers. The incorporation of these distinctions in a more sophisticated description of the lipid solution may be expected to reproduce further detailed properties of real 
systems.

In summary, we have extended the MLG framework to include the solvation of amphiphilic solutes in water. Within a cubic HP model we have found the aggregation of solute particles, and the formation of various types of micelle as a function of the distribution of hydrophobic regions. By successive substitution of hydrophobic sides by polar ones, we have studied the aggregation behavior and the influence of the degree of hydrophobicity on the upper and lower critical solution temperatures. We have refined this model to describe lipid molecules of varying length, by adapting the interaction of the hydrophobic tail to include a corresponding number of neighboring water molecules, and have demonstrated the enhanced stability of aggregates with increasing tail length (increasing hydrophobicity). We have shown that primary features of micelle formation, which are often attributed solely to the amphiphilic nature of the solute particles under consideration, are reproduced by our extension of the solvent-based MLG model to describe alterations of water structure in the vicinity of the different surface regions of dissolved amphiphiles.

\section{Acknowledgments}

We are grateful to the Swiss National Science Foundation for financial support through grants FNRS 2161397.00 and 2000-67886.02.
[1] R. Gomes de Azevedo, L.P.N Rebelo, A.M. Ramos, J. Szydlowski, H.C. de Sousa, and J. Klein, Fluid Phase Equilibria 185, 189 (2001).

[2] L.P.N. Rebelo, Z.P. Visak, H.C de Sousa, J. Szydlowski, R. Gomes de Azevedo, A.M. Ramos, V. NajdanovicVisak, M. Nunes da Ponte, and J. Klein, Macromolecules 35, 1887 (2002).

[3] F.H. Stillinger and A. Ben-Naim, J. Chem. Phys. 74, 2510 (1981).

[4] L. Leibler, H. Orland, and J.C. Wheeler, J. Chem. Phys. 79, 3550 (1983).

[5] J.N. Israelachvili, D.J. Mitchell, and B.W. Ninham, J. Chem. Soc. Faraday Trans. II 72, 1525 (1976).

[6] C. Tanford, The Hydrophobic Effect (Wiley, New York, 1980).

[7] L.-J. Chen, S.-Y. Lin, and C.-C. Huang, J. Phys. Chem. B 102, 4350 (1998).

[8] J.C. Lang and R.D. Morgan, J. Chem. Phys. 73, 5849 (1980).

[9] W. Wenzel, C. Ebner, C. Jayaprakash, and R. Pandit, J. Phys.: Condens. Matter 1, 4245 (1989).

[10] H. Wennerström and B. Lindman, Phys. Rep. 52, 1 (1979).

[11] G.J.T. Tiddy, Phys. Rep. 57, 1 (1980).

[12] R.E. Goldstein, J. Chem. Phys. 84, 3367 (1986).

[13] M. Corti, L. Cantu, and P. Salina, Adv. Colloid Interface Sci. 36, 153 (1991).

[14] R.G. Larson, J. Chem. Phys. 96, 7904 (1992).

[15] G. Gompper and M. Schick, Self-assembling amphiphilic systems (Academic Press, London, 1994).

[16] A. Bhattacharya and S.D. Mahanti, J. Phys.: Condens. Matter 12, 6141 (2000).

[17] A. Bhattacharya and S.D. Mahanti, J. Phys.: Condens. Matter 13, L861 (2001).

[18] A. Milchev, A. Bhattacharya, and K. Binder, Macromolecules 34, 1881 (2001).

[19] W. Kauzmann, Adv. Protein Chem. 14, 1 (1959).

[20] N. Muller, Acc. Chem. Res. 23, 23 (1990).
[21] B. Lee and G. Graziano, J. Am. Chem. Soc. 22, 5163 (1996).

[22] S. Moelbert and P. De Los Rios, Macromolecules 36, 5845 (2003).

[23] S. Moelbert and P. De Los Rios, J. Chem. Phys. 119, 7988 (2003).

[24] P.L. Privalov and S.J. Gill, Adv. Protein Chem. 39, 191 (1988).

[25] P.H.K. De Jong, J.E. Wilson, and G.W. Neilson, Mol. Phys. 91, 99 (1997).

[26] A. Perstemilidis, A.M. Saxena, A.K. Soper, T. HeadGordon, and R.M. Glaeser, Proc. Natl. Acad. Sci. USA 93, 10769 (1996).

[27] F.H. Stillinger, Science 209, 451 (1980).

[28] A. Ben-Naim, Water and Aqueous Solutions: Introduction to a Molecular Theory (Plenum Press, New York, 1974).

[29] G. Némethy and H.A. Scheraga, J. Chem. Phys. 36, 3401 (1962).

[30] R. Ludwig, Angew. Chem. Int. Ed. 40, 1808 (2001).

[31] K.A.T. Silverstein, A.D.J. Haymet, and K.A. Dill, J. Chem. Phys. 111, 8000 (1999).

[32] Y. Kita, T. Arakawa, T.-Y. Lin, and S.N. Timasheff, Biochemistry 33, 15178 (1994).

[33] P. De Los Rios and G. Caldarelli, Phys. Rev. E 62, 8449 (2000).

[34] G. Caldarelli and P. De Los Rios, J. Biol. Phys. 27, 229 (2001).

[35] P. De Los Rios and G. Caldarelli, Phys. Rev. E 63, 031802 (2001).

[36] S. Moelbert and P. De Los Rios, cond-mat/0305204 (unpublished).

[37] K.A.T. Silverstein, A.D.J. Haymet, and K.A. Dill, J. Am. Chem. Soc. 122, 8037 (2000).

[38] Yalkowsky, S.H. Solubility and Solubilization in Aqueous Media (American Chemical Society and Oxford University Press, New York, 1999). 